\title{
Cluster galaxies 10 billion years ago
}

\author{
V. Strazzullo ${ }^{1}$, E. Daddi ${ }^{1}$, R. Gobat ${ }^{1}$, and M. Onodera ${ }^{2}$ \\ ${ }^{1}$ CEA-Saclay, 91191 Gif sur Yvette, France ${ }^{2}$ ETH Zürich, 8093 Zürich, Switzerland \\ email: veronica.strazzullo@cea.fr
}

\begin{abstract}
Cl} \mathrm{J} 1449+0856$ is a spectroscopically confirmed galaxy cluster at $z \sim 2$. The detection of a faint, extended X-ray emission, suggestive of an already evolved, partially relaxed structure, puts this system among the most distant "established" clusters rather than in the realm of $z \gtrsim 2$ proto-clusters. This gives us a chance of studying galaxies in an evolved overdense environment very close to their formation epoch, and in particular to trace the evolution of early-type galaxies in clusters back to ten billion years ago.
\end{abstract}

Keywords. Galaxies: clusters, Galaxies: evolution, Galaxies: high-redshift

$\mathrm{Cl} \mathrm{J} 1449+0856$ at $z=2$ is the most distant X-ray detected and spectroscopically confirmed galaxy cluster discovered so far. We studied its galaxy populations with a sample of $\sim 100$ candidate cluster members $\left(\gtrsim 10^{10} \mathrm{M}_{\odot}\right)$ within $500 \mathrm{kpc}$ of the cluster center. Already at this redshift, the central cluster region $(r<200 \mathrm{kpc})$ hosts a population of massive galaxies with quiescent stellar populations and early-type morphologies. At the same time, the cluster core also seems to host massive galaxies still in their active formation phase, with significant star formation and ongoing merging activity. A clear correlation is observed between morphological structure and stellar populations, with quiescent galaxies typically showing a high Sersic index profile, as also observed in the field up to this redshift. In general agreement with many high-redshift studies, quiescent early-type candidate cluster members are smaller than similarly massive early-types in the nearby Universe, although the most compact $z \sim 2$ galaxies in our field are spectroscopically confirmed interlopers, leaving the mass-size relation of cluster early-types a factor 2-3 lower in size than the commonly used Shen et al. (2003) local determination.
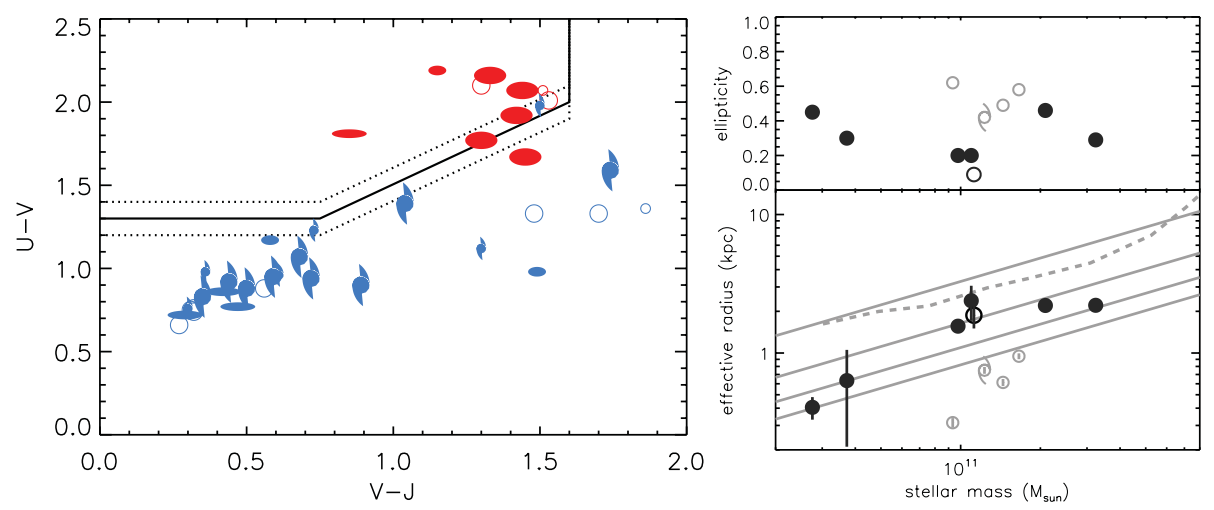

Figure 1. Left: UVJ restframe color plot (Wuyts et al. 2007) of candidate cluster members $\left(\mathrm{m}_{F 140 W}<24.5\right.$, symbol shape according to Sersic index $)$. Right: effective radius and ellipticity vs stellar mass for candidate quiescent early-type cluster members (dark symbols), and for 4 quiescent spectroscopic interlopers at similar redshift (light symbols). Solid lines show the Shen et al. (2003) local relation scaled down in size by a factor $1,2,3,4$. 